\title{
« Les modalités d'intégration des PGI (Progiciels de Gestion Intégrés) dans la structure organisationnelle: Cas d'une PME Marocaine »
}

\author{
Siham JABRAOUI \\ Laboratoire de recherche ISO Ecole Nationale de Commerce et de Gestion de Casablanca ENCG-C \\ Université Hassan II de Casablanca- Maroc
}

\begin{abstract}
Résumé : Ce que l'on appelle intégration des progiciels de gestion intégrés (PGI), conduit généralement à une refonte du système d'information de gestion, et à une remise à plat des procédures de gestion en place, lesquelles peuvent être par la suite réorganisées suivant les orientations stratégiques ciblées par l'entreprise. Les principaux problèmes commencent lorsqu'il s'agit d'intégrer les fonctionnalités spécifiques à l'entreprise dans le PGI. Entre la standardisation des processus de gestion et la personnalisation du PGI, les entreprises semblent être souvent en quête d'un équilibre qui reflète la cohérence de leurs choix et de leurs décisions avec leurs orientations stratégiques. C'est sur ce thème que notre présente recherche se positionne. Elle vise dans ce cadre à explorer les différents choix stratégiques effectués par une PME Marocaine pour intégrer un PGI. C'est sur la base de ces choix que nous avons tenté de décrypter les différentes modalités adoptées pour intégrer ces progiciels.
\end{abstract}

Abstract : What is called integration of Enterprise Resource Planning (ERP) usually leads to a rebuilding of management information systems, and to a revisit of management procedures that can be thereafter reorganized according to the company's strategic orientations. The main problems start when one wants to integrate specific functionalities of a particular company into the ERP. Between the standardization of management procedures and the personalization of ERP, companies often seem to look for a balance that reflects the coherence of their choices and decisions according to their strategic orientations. It's this topic of balance which constitutes the heart of this dissertation. This research aims to explore the various strategic choices done by Morrocan SME to integrate ERP. It's on the basis of these choices that we have tried to decrypt the different modalities adopted to integrate these softwares.

Mots clés: Changement organisationnel, Intégration organisationnelle, Management stratégique, PME, Progiciel de Gestion Intégré (PGI).

Key words: Enterprise Resource Planning(ERP), Organisationnel Change, Organisationnel Integration, SME, Strategic Management.

\section{INTRODUCTION}

Les projets de mise en place des systèmes d'information en général et des PGI (Progiciels de Gestion Intégrés) en particulier sont généralement des projets lourds, complexes et risqués (Vincent et Gharbi, 2003 ; Bernard, Rivard, Aubert, 2004) [1]. Ce que l'on appelle l'«implémentation » de ces progiciels constitue une refonte du système d'information de gestion (Mourlon et Neyer, 2002) [2], et nécessite généralement une remise à plat des procédures de gestion en place (Bancroft, 1996 ; Brynjolfsson et Hitt, 2000; Gu et Gera, 2004; Dostie et Jayaraman, 2008) [3] qui peuvent être par la suite réorganisées (Lequeux, 1999) [4] suivant les orientations stratégiques ciblées par l'entreprise (El Amrani, 2003) [5]. Les fournisseurs proposent pour cela des fonctionnalités considérées comme riches et éprouvées, voire fondées sur les meilleures pratiques (Baile et Lesuisse, 2002) [6].

Les principaux problèmes commencent lorsqu'il s'agit d'intégrer les fonctionnalités spécifiques à chaque entreprise dans le PGI, qu'il s'agisse d'entreprises opérant dans le même secteur d'activité ou dans des secteurs différents. L'une des préoccupations centrales de ces entreprises consiste éventuellement à savoir si le système en question est capable de couvrir la majorité de leurs besoins fonctionnels de base dans le respect de leur orientation stratégique globale. Ces entreprises se posent également la question relative aux possibilités de paramétrage proposées par ces progiciels et leur capacité de répondre aux besoins spécifiques à chaque entreprise (Hanseth et Braa, 1998 ; Watson et al., 1999) [7]. Entre la standardisation des processus de gestion et la personnalisation du PGI, les entreprises semblent être souvent en quête d'un équilibre qui reflète la cohérence de leurs choix et décisions avec leurs orientations stratégiques et qui leur assure des résultats performants.

Pour cela, notre recherche vise à apporter de l'éclairage sur la question de l'intégration des PGI. En effet, l'environnement des entreprises est caractérisé par une complexité accrue, due essentiellement à un rythme d'évolution accéléré, à la particularité des problèmes liés à la transversalité des processus, à la difficulté de leur 
intégration, à un besoin d'adaptation permanent des solutions informatiques aux spécificités des entreprises ou inversement...

Cette recherche vise à explorer principalement les différents choix stratégiques effectués par une entreprise industrielle marocaine pour intégrer un PGI et les impacts de ces choix sur le plan organisationnel. C'est sur la base de ces choix que nous tenterons de décrypter les différentes modalités adoptées pour intégrer ces progiciels et d'analyser leurs différents impacts organisationnels. Il s'agit d'une étude rétrospective d'un projet d'intégration d'un PGI dans le cas d'une PME industrielle. L'évaluation de ce projet d'intégration nous permettra de construire une représentation pragmatique du phénomène de l'intégration, de situer la ou les méthode(s) d'intégration adoptée(s) et d'étudier leurs impacts organisationnels.

\section{LE PGI : OBJET D’INTEGRATION ORGANISATIONNELLE}

Dans un environnement complexe caractérisé par une concurrence accrue, les entreprises cherchent en permanence l'amélioration de leur situation financière, technologique et organisationnelle. Toutefois, elles visent à être à jour par rapport aux concurrents, si ce n'est à disposer d'un avantage concurrentiel par rapport à ces derniers. Dans une telle course à la performance, ces entreprises cherchent à disposer des dernières armes technologiques (Gu et Gera, 2004, Dostie et Jayaraman, 2008) [8]. Elles sont amenées par ailleurs à réorganiser leur système d'information de manière générale ou partielle.

En effet, les exigences de l'environnement concurrentiel dans lequel elles opèrent les incitent à disposer des moyens nécessaires pour pouvoir s'adapter, se renouveler et innover. Le SI est l'un des outils fondamentaux qui aident ces entreprises à être réactives et à fournir des réponses concrètes à leurs besoins fonctionnels au niveau interne et externe. Face à ces impératifs, les systèmes d'information composés de solutions informatiques hétérogènes et cloisonnés par fonction ont montré leurs limites. Ils sont incapables de fournir aux entreprises en temps réel les informations nécessaires à une gestion intégrée de leurs chaînes de valeur. Chose que peut leur procurer les progiciels de type PGI. Ces solutions ont permis grâce au progrès technologique en matière des technologies d'information, de faire communiquer l'ensemble des fonctions d'une entreprise en temps réel et via une base de données unique.

Un PGI peut être défini comme un ensemble d'outils informatiques intégrés modulaires et paramétrables (Reix, 2000) [9] qui sert à contrôler les ressources qui circulent dans l'organisation (Bancroft et al 1997, Davenport 1998, Granlund et Malm 2000) [10]. Un ensemble de modules correspondants chacun à un processus de gestion -approche horizontale- ou à une fonction- approche verticale-. Chaque module est composé essentiellement de deux grandes parties: une partie de base standard et une partie personnalisable- La partie standard représente un référentiel unique qui reprend les règles de gestion standards, appelées également: best practices. Ces meilleures pratiques sont identifiées, recensées et choisies sur la base des retours d'expériences des clients et des intégrateurs des PGI lors des phases de choix, de déploiement et de fonctionnement du système et même après. Baile et Lesuisse (2002) [11] qualifient cette pratique de «Processus d'enrichissement progressive des fonctionnalités d'un logiciel ». Ils considèrent que la construction d'un tel logiciel générique (qui a des usages multiples ou qui peut être utilisé dans divers contextes organisationnels) demande un temps de développement qui doit au moins dépasser une dizaine d'année. - La partie personnalisable permet une certaine adaptation de l'architecture applicative aux besoins spécifiques de l'entreprise, à travers le paramétrage (Watson et al, 1999) [19]. Cependant, le degré d'adaptabilité ne peut dépasser un certain seuil. La spécificité des PGI qui réside dans leur proposition d'un modèle standardisé des processus de gestion est susceptible de diminuer ce degré d'adaptabilité (Hanseth et Braa, 1998) [13]. Cela peut d'ailleurs constituer un indicateur de flexibilité d'un PGI par rapport à un autre, en comparant les possibilités de paramétrage proposées par chacun pour s'adapter à un besoin donné.

La question qui se pose à ce niveau est : jusqu'à quel point un PGI peut-il s'adapter à une organisation à travers le paramétrage ? Autrement dit, jusqu'à quel point l'architecture organisationnelle d'une entreprise peut-elle être impactée par l'offre architectural proposée par le standard PGI? La réponse à cette question du point de vue des éditeurs et des clients n'est pas vraiment la même. D'un côté, l'idéal pour un client consiste généralement à avoir un PGI flexible et adaptable, avec de grandes possibilités de paramétrage. Toutefois, ce n'est pas toujours la règle chez tous les clients. Tout dépend, en fait, des objectifs stratégiques de ces entreprises : veulent-elles se restructurer autour de la solution standard, ou préserver leurs organisations et faire adapter la solution?

Du point de vue de l'éditeur, tout PGI offre des possibilités de paramétrage et d'adaptabilité. Néanmoins, ces adaptations ne peuvent aller jusqu'à modifier le cœur applicatif de la solution, c'est à dire les processus de fonctionnement. En revanche, tout ce qui est accessoire et esthétique au système est paramétrable, mais là aussi dans une certaine limite. 
L'objectif de la présente recherche c'est d'apporter de la lumière sur la démarche stratégique adoptée par une entreprise industrielle intégrant un PGI et l'impact de cette intégration sur la structuration organisationnelle de cette entreprise.

\section{APPROCHE SYSTEMIQUE DE LA COMPLEXITE D'INTEGRATION DES PGI}

Le concept de la complexité existe depuis toujours mais sa prise de conscience de manière scientifique est récente. Ce qui explique la lente émergence de la systémique en tant qu'approche théorique pratique et méthodologique relatives à l'étude de ce qui est reconnu comme trop complexe pour pouvoir être abordé de façon réductionniste ( AFSCET , 2003 ; Donnadieu et al.2003) [14].

Sans complexité, le rationalisme analytique pouvait être par ailleurs suffisant pour appréhender le concept de l'intégration des PGI. Ce concept renvoie aux difficultés de compréhension révélées par les acteurs eux-mêmes. Se lancer dans un projet PGI, «est une aventure trop risquée » tel est le ressenti des acteurs projets PGI rencontrés à l'occasion de cette recherche. Il y a toujours du flou, de l'incertain, de l'imprévisible, de l'ambiguë et de l'aléatoire dans ce type de projet et c'est ce qui qualifie les situations complexes selon Lapointe (1992) [15]: «Elles sont floues, changeantes et peu structurées. Elles peuvent être étudiées sous différentes perspectives, sous différents angles, en fonction de plusieurs structures cognitives et de divers systèmes de valeurs ». L'entreprise a beau fournir des efforts considérables au niveau de la communication, de l'information et de l'accompagnement des acteurs et des utilisateurs. Malgré cela, elle n'arrive pas à tout maîtriser. Ce phénomène de complexification qui entoure l'intégration des PGI nous amène à l'explorer à travers des approches qui nous paraissent capables de combler certaines lacunes ou insuffisances de l'approche expérimentale. Les préceptes réductionnistes de la réalité telle qu'elle est envisagée par cette approche expérimentale justifient les doutes émis par plusieurs scientifiques (Checkland 1976, 1981 ; Commoner, 1972 ; LeMoigne 1977, Watzlawick 1980) [16] sur son efficacité dans l'étude des systèmes complexes.

Selon la démarche systémique, l'approche globale consiste à aborder tous les aspects d'un problème progressivement mais non séquentiellement. Elle permet d'exprimer à la fois l'interdépendance des éléments du système et la cohérence de l'ensemble (LeMoigne, 1977) [17]. Nous pouvons ainsi considérer que la présente recherche part d'une vue générale et globale du concept de l'intégration des PGI dans l'organisation. L'approfondissement des détails de la relation système d'information et système organisationnel s'effectuera dans l'objectif d'expliciter la nature de ces relations à la fois au niveau local des fonctions et au niveau global de l'entreprise. L'intégration organisationnelle est un concept qui est à notre sens fédérateur de plusieurs notions différentes mais non contradictoires. Il regroupe l'ensemble des sous-systèmes: informationnel et organisationnel, ainsi que les environnements qui les entourent (Le Moigne, 1977). Cette décomposition de l'organisation n'est pas une fin en soi (Bertalanffy, 1956) [18]. Elle vise à étudier le phénomène de l'intégration en l'analysant en profondeur au niveau séquentielle des entités fonctionnelles avant de transposer l'analyse au niveau global de l'entreprise. Elle nous permettra sans doute de mieux articuler les sous systèmes : PGI et organisation dans une seule problématique. Toutefois, l'intégration du système informationnel de type PGI dans le système organisationnel nécessite un approfondissement des relations qu'entretiennent ces sous-systèmes sur la base d'un certains nombres de paramètres pertinents, choisis selon la finalité de la recherche. Dans la réalité, les systèmes existent à travers le construit théorique qu'on leur attribue. Pour Morin(1982) [19], les systèmes n'existent qu'à travers les yeux de ceux qui les voient. Pour notre recherche, un système existe dans les relations qu'il entretient avec les autres systèmes. Ceci nous incite à qualifier l'intégration du système informationnel dans le système organisationnel d'une sorte de reconstruction des deux systèmes à travers les interactions de leurs composants dans un schéma cible global. Jean luis Le Moigne (1994) [20] propose une théorie de la modélisation d'un système complexe adaptée du modèle à neuf niveaux de complexité de Boulding (1956) [21]. Cette modélisation à complexité croissante propose une représentation théorique adaptable à tout système. Ce modèle a également été repris par Bertalanffy (1968) [22]. Ce modèle peut tout à fait s'appliquer au projet d'intégration des PGI en considérant ce dernier comme un sous système (informationnel) d'un système global (système organisationnel) et qui co-existe avec d'autres sous systèmes, en l'occurrence le système décisionnel et le système opérationnel. 


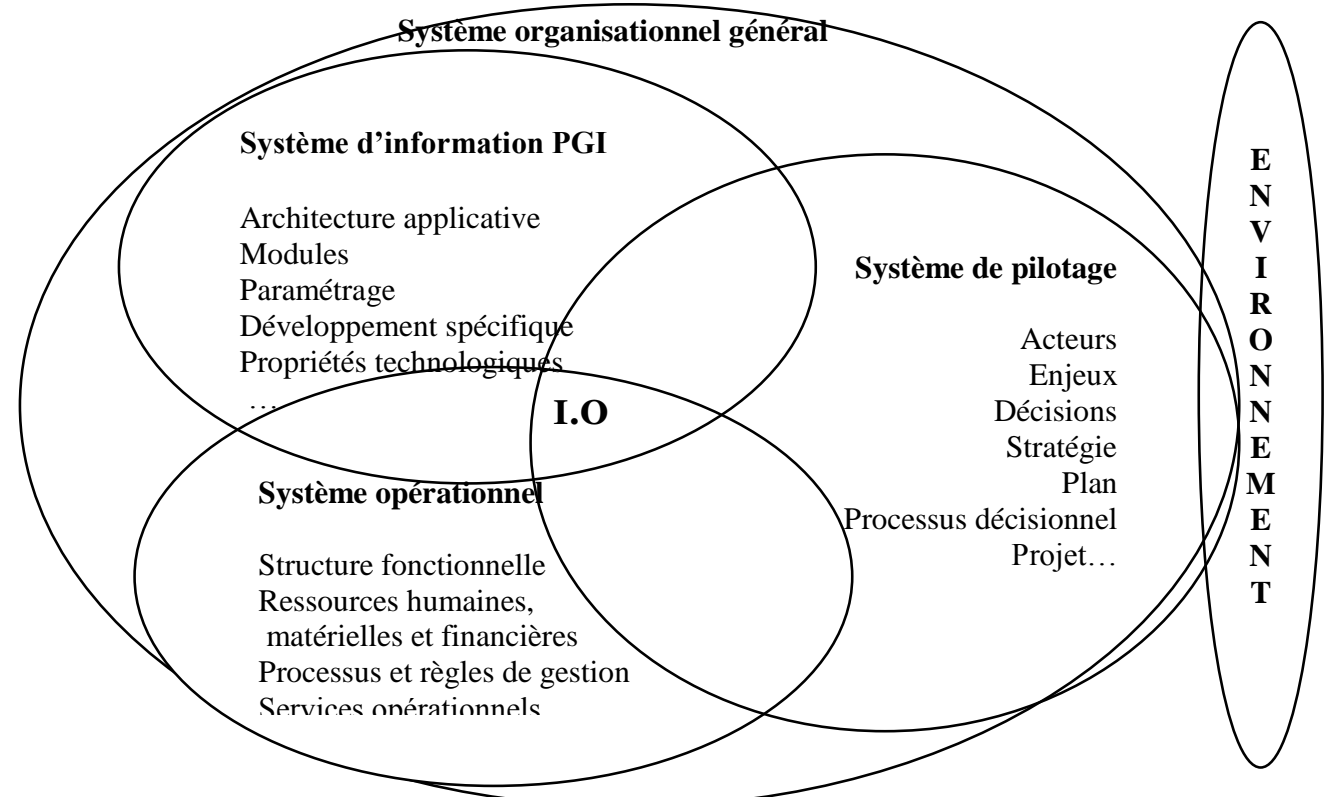

Figure 1 : Modélisation systémique de l'intégration organisationnelle des PGI

Selon le modèle proposé, l'intégration organisationnelle (I.O) des PGI se situe à l'intersection des trois sphères qui composent le système organisationnel d'une entreprise : la sphère informationnelle, la sphère opérationnelle et la sphère décisionnelle. Le concept de l'intégration peut être défini par ailleurs comme un ensemble d'interactions permanentes des variables organisationnelles et technologiques (Deltour, Vaast 2000) [23]. Il s'agit d'une construction partagée, basée sur l'interdépendance des éléments du système d'information de l'entreprise (le PGI en l'occurrence) et les éléments de ses systèmes ; opérationnel et décisionnel. La variable environnementale est considérée au même titre que les autres variables comme explicative de la typologie d'intégration organisationnelle adoptée par les entreprises dont le système organisationnel est de nature interdépendant avec son environnement selon l'approche systémique (Bertalanffy,1973) [24].

\section{L'INTEGRATION DES PGI ET LE MANAGEMENT STRATEGIQUE DES PROJETS SYSTEMES D'INFORMATION}

Comme on vient d'analyser, les projets d'intégration des PGI sont des projets complexes, importants du point de vue des investissements financiers et humains qu'ils demandent, et très risqués du point de vue de leur succès et de leur retour sur investissement. Sans une véritable démarche stratégique, claire et transparente, sans une démarche de mise en place justifiée et en cohérence avec les orientations stratégiques de l'entreprise, le projet peut prendre une tournure non prévisible, et des résultats non anticipés peuvent mettre la vie du projet et de l'entreprise en péril. Le manque de visibilité des entreprise sur les vrais repères méthodologiques du « comment intégrer un PGI ?» les pousse à se réfugier dans les expériences préalables d'autre entreprises sur des projets similaires, ou de se contenter de recourir à l'assistance externes des consultants, intégrateurs...

L'étude de la question de l'intégration des PGI et ses implications organisationnelles relève naturellement du domaine du management stratégique «L'intégration des impacts et des opportunités des TIC dans le management des entreprises s'applique bien naturellement aux questions stratégiques » (Kalika 2003) [25]. Le traitement de cette question du point de vue du management stratégique permet surtout de l'approcher selon une démarche stratégique basée sur le diagnostic, le choix et le déploiement dans la recherche d'une cohérence d'ensemble entre la stratégie SI et la stratégie globale de l'entreprise. Notre propos à ce niveau est de positionner cette démarche au cœur de la problématique de l'intégration organisationnelle et de faire une relecture des composantes de la relation organisation-PGI d'un point de vue stratégique.

Le management stratégique nous a procuré des concepts clés qui se sont naturellement imposés dans l'étude de l'articulation entre PGI et organisation sous la thématique de l'intégration organisationnelle. Il représente pour notre recherche un support méthodologique qui permet d'élucider cette question d'intégration en plaçant la démarche stratégique du choix et du déploiement des PGI au centre de l'analyse des pratiques d'intégration et en considérant le PGI en tant qu'élément interdépendant, co-constructeur de la stratégie (Rallet, 1992 ; Daft et Lengel, 1986) [26] et moyen de sa mise en place (Davis, 1974) [27]. A travers la mobilisation de ce cadre, nous tentons de mettre en évidence l'intérêt de plusieurs variables que nous avons jugées pertinentes. La vision cible (Swanson et Ramiler, 1997 ; El Amrani et al., 2002) [28] est l'une de ces variables dont l'analyse s'est avérée 
primordiale pour la compréhension du processus du choix et de décision relatif au projet PGI. Elle permet en interférence avec les variables qui qualifient l'environnement de la décision relative au choix de l'approche intégrée, au choix de la solution PGI, à l'analyse et la gestion des écarts stratégiques, de concrétiser la vision que peut avoir une entreprise de son organisation cible émergeante du croisement d'un ensemble de paramètres organisationnels, stratégiques et techniques. Elle peut ainsi être considérée comme un facteur critique de succès d'un projet PGI (El Amrani et al., 2002) [29] et source d'innovation organisationnelle (Senge, 1990) [30] dans la mesure où elle fournit à l'entreprise une représentation synthétique des grandes lignes stratégiques qui vont conditionner la suite du déroulement du projet et serviront à piloter les actions de changement.

La notion d'écart stratégique (Chang et Gable, 2001 ; Kale, 2000 ; Lyytinen et al, 1998 ; Soh et al, 2000) [31] entre Les besoins de l'entreprise en matière des SI et les propositions de la solution avant de se lancer dans l'implémentation fait partie des variables que nous avons retenues pour justifier l'adoption de différentes démarches d'intégration organisationnelle du PGI au sein de la même entreprise.

Dans un projet PGI caractérisé par sa complexité, l'efficacité d'une décision relative par exemple au choix de la solution se situe en amont de son développement. Nous décidons par ailleurs de prendre en compte la notion «d'analyse des écarts » dans le processus de choix de la solution afin de mettre en valeur le rôle du contrôle stratégique des décisions prises à CT dans l'augmentation de l'efficacité des actions engagées au cours du projet. Ainsi, lorsque de gros écarts surgissent de la confrontation d'une solution PGI aux besoins d'une entreprise, la cohérence stratégique est synonyme dans ce cas d'écartement de cette solution et de la recherche d'une autre plus proche des aspirations de l'entreprise. Lorsqu'une entreprise décide de choisir une solution en dépit des écarts constatés, d'autres facteurs peuvent entrer en jeux pour justifier cette incohérence stratégique. Il s'agit essentiellement des rapports de force que peut subir hiérarchiquement cette entreprise de sa société mère qui lui impose son PGI, de son intégrateur qui peut l'influencer dans un choix (même s'il est incohérent), d'un directeur général ou d'un directeur de projet qui ne se fait qu'à sa tête...etc.

L'intégration des PGI d'un point de vue processus intègre des paramètres qui se rattachent particulièrement aux caractéristiques du projet PGI : coûts, délais, acteurs, périmètre fonctionnel...ces caractéristiques ont à notre sens un impact sur la démarche d'intégration adoptée par l'entreprise qui intègre un PGI.L'intégration organisationnelle des PGI est un compromis entre les besoins exprimés par l'entreprise et les solutions proposées par le PGI. Ce compromis consiste à faire un arbitrage entre paramétrage de la solution et développements spécifiques. C'est sur la base de cet arbitrage que se dessine la logique dominante de la démarche d'intégration adoptée. C'est pourquoi nous avons choisi de considérer cet arbitrage comme une variable de différenciation entre les différentes méthodologies d'intégration adoptées dans le cas de l'entreprise qui fait l'objet de la présente recherche.

L'intégration organisationnelle des PGI en tant que concept théorique visant la cohérence d'ensemble entre PGI et organisation est appréhendée opérationnellement grâce également à la mobilisation du concept de l'alignement stratégique (Henderson et Venkaraman, 1993 ; Luftman, 1996 ; Chan et Huff, 1993 ; Reich et Benbasat, 1996) [32]. Dans le cadre d'un projet d'implémentation de PGI, « le management stratégique des technologies d'information » qui est une discipline émergente au sein des sciences de gestion (Venkatraman, 1995) [33] s'articule essentiellement sur le positionnement de la technologie et son alignement sur le système organisationnel (Vaujary, 2002) [34]. Il stipule ainsi la formulation stratégique à deux niveaux (Marchesnay, 2004) [35] : Le premier concerne l'ensemble de l'organisation (Corporate), le second concerne la formulation au niveau (Business) des entités fonctionnelles. Dans cette perspective, les stratégies développées au niveau des entités peuvent dévier ou s'opposer à celles tracées au niveau Corporate. Mais tant que cette déviation ne remet pas en cause le bon déroulement du projet, il est recommandé de laisser le maximum d'initiative stratégique aux divisions ou entités opérationnelles (Marchesnay, 2004). Cette perspective ne devient véritablement objective qu'après vérification des synergies possibles. La « synergie » est définie par Ansoff (1965) [36] comme la règle du $(2+2=5)$ : les activités additionnelles viennent renforcer la compétitivité des activités existantes. Dans le cadre de l'intégration des PGI, les démarches d'intégration adoptés par les différentes entités fonctionnelles de l'entreprise peuvent diverger du cadre général assigné au projet (imposé ou proposé soit par l'entreprise soit par la solution elle même). Ainsi, chaque entité peut adopter une démarche qui répond bien à ses attentes et ses besoins. La synergie de ces différentes démarches doit s'inscrire dans un schéma général cohérent. Notre choix d'adoption du management stratégique comme support méthodologique pratique et opérationnel provient également de notre volonté de rapprocher un certain nombre de concepts avérés essentiellement sur le plan théorique et moins reconnus voir mal connus sur le plan pratique.

Nous reprenons dans la figure qui suit ce qui a été analysé précédemment à travers ce schéma conceptuel : 


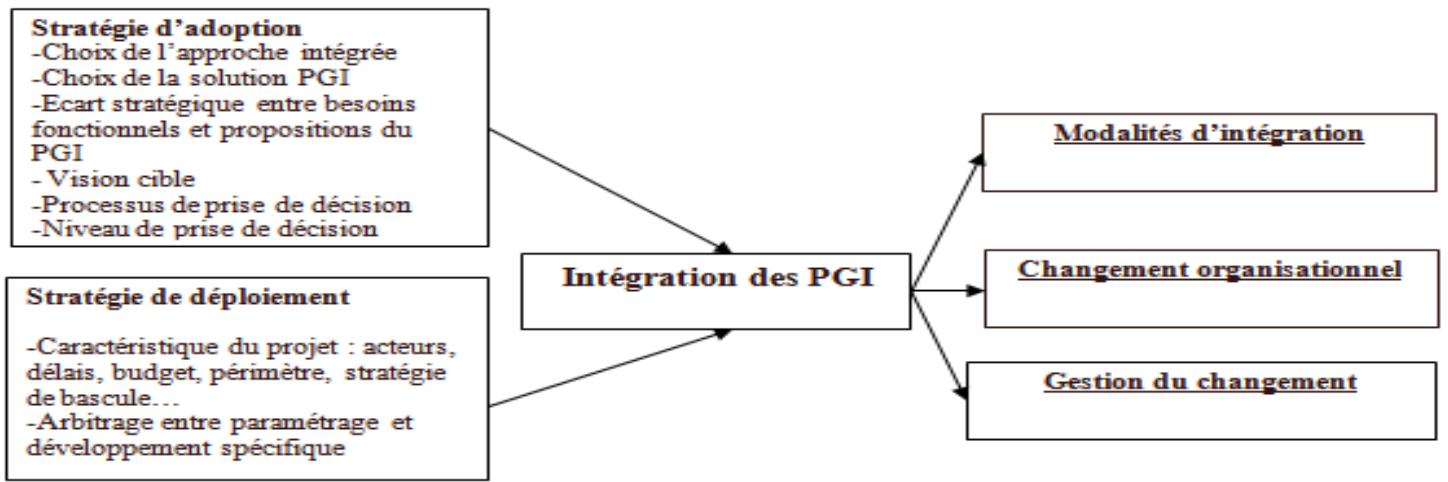

Figure 2 : Schéma conceptuel de la recherche

\section{LES MODALITES D'INTEGRATION DES PGI DANS LA STRUCTURE ORGANISATIONNELLE : CAS D'UNE PME MAROCAINE}

Cette recherche s'inscrit dans une perspective compréhensive. Elle vise à étudier l'intégration des PGI en tant que phénomène organisationnel qui implique toute les composantes d'une entreprise ou presque. Elle vise particulièrement à formuler une représentation explicite des modalités d'intégration adoptées par une PME à l'occasion d'un projet PGI et les implications de ce phénomène au niveau organisationnel.

Pour ce faire nous recourons à une étude de cas d'une PME Marocaine (Alpha) qui opère dans le secteur industriel (la sidérurgie). Nous considérons que le choix de cet instrument est justifié dans la mesure où il répond aux objectifs de la recherche, à savoir : accéder aux faits, aux représentations et aux interprétations sur des situations connues par les acteurs concernés par le projet PGI (Wacheux, 1996). En effet, l'étude de cas permet d'analyser en profondeur le contexte du projet PGI, les motifs du choix de la solution, les démarches du déploiement de la solution dans les différentes fonctions de l'entreprise, ainsi que les implications de ces choix sur le plan organisationnel en général. L'entreprise Alpha exploitait son PGI «SAP» depuis 4 ans. Elle a choisi d'intégrer les modules fonctionnels suivants : FI(Finance), SD (ventes et distribution), MM (gestion des achats), HR (gestion des ressources humaines), PP (gestion de la production), CO (contrôle de gestion). Nous avons mené notre enquête qualitative via des entretiens semi-directifs. Nos principaux interlocuteurs nous ont permis d'avoir une idée globale sur le déroulement du projet PGI et ses implications organisationnelles sur cette entreprise. Nous avons par ailleurs fait le choix de croiser les visions des différents acteurs concernés par le projet PGI à savoir: La vision de la direction SI(2), celle de la direction générale(1), celle de l'équipe projet PGI(1) et celle des utilisateurs du système (5).L'interprétation des résultats de cette étude nous ont permis de construire une représentation pragmatique du phénomène de l'intégration des PGI et des résultats de ce projet sur le plan organisationnel. Nous avons par ailleurs l'ambition d'inscrire notre recherche dans une perspective interprétativiste avec une visée compréhensive.

\section{L'intégration d'un PGI dans une PME Marocaine}

L'intégration organisationnelle des PGI est à notre sens une variable dépendante de plusieurs variables (Fig 2). Notre objectif dé le départ consiste à analyser la façon avec laquelle ces variables contribuent à l'explication des différentes logiques d'intégration qui peuvent exister. Nous avons donc insisté sur l'implication stratégique que peut avoir chacune des variables sur le phénomène étudié.

Chaque projet PGI est incontestablement le résultat d'une décision stratégique. Nous avons voulu comprendre comment l'entreprise en question a décidé d'adopter une approche PGI et comment elle a procédé pour choisir son PGI. Pour préparer le projet, l'entreprise connaît sans doute ses besoins et ses objectifs stratégiques. Notre but était de savoir si l'entreprise en question a bien défini l'organisation qu'elle ciblait en dehors du PGI ou à posteriori, et quelles étaient les implications de cette décision sur le déroulement du projet.

Après avoir analysé les implications des décisions stratégiques en termes de processus de prise de décision et du niveau de prise de décision, notre but était de chercher la nature de la relation qui peut y avoir entre la stratégie d'adoption et celle de mise en place. Cette articulation nous a permis par la suite de voir plus claire le positionnement de la démarche d'intégration ainsi que ses implications organisationnelles sur les plans financier humain et processus internes

\subsection{Le choix de la solution PGI}

La démarche de choix de la solution peut avoir des répercussions sur la bonne marche du projet. Il s'agit pour l'entreprise de trouver la solution qui couvre ses besoins opérationnels en prenant en compte ses 
spécificités fonctionnelles. A ce titre, les entreprises sont amenées à faire très attention à la phase d'analyse des besoins notamment les plus spécifiques d'entre eux. C'est une étape primordiale dont dépendent tous les processus de choix et de mise en place de la solution. Elle exige de l'entreprise un travail de mise à plat des procédures, de formulation des besoins généraux et spéciaux, une parfaite connaissance des métiers et de leurs spécificités et une bonne connaissance des solutions existantes sur le marché de l'édition. Une confrontation «test»des besoins aux propositions fonctionnelles des solutions peut ainsi donner une idée générale sur la couverture la plus proche de ce que souhaite avoir l'entreprise. Généralement le choix d'une solution PGI peut suivre une démarche classique : de formulation de cahier de charge, ensuite l'établissement d'une schort list avant la prise de décision finale; tel était le cas de notre PME dans cette recherche. Cette entreprise reconnait avoir procédé selon une démarche classique de formulation de cahier de charge détaillé qui intègre l'ensemble des besoins analysés lors de la phase avant projet. Toutefois, l'entreprise n'a fait de prospection qu'auprès d'un seul éditeur puisqu'elle avait déjà une idée préalable de la solution qu'elle veut implémenter et qui lui a été fortement conseillée par ses partenaires. Cette idée a été réconfortée par la présentation faite sur site d'un commercial de la société éditrice. Le choix d'une solution PGI peut dans certains cas suivre une démarche de développement participatif c'est-à-dire que l'entreprise accepte de faire l'objet d'une expérimentation d'une nouvelle solution à des conditions attirantes en termes de coûts.

\subsection{La démarche stratégique d'adoption des PGI}

Nous analysons à ce niveau l'ensemble des paramètres que nous avons jugés pertinents pour qualifier la démarche adoptée par l'entreprise pour adopter son PGI à savoir : le choix d'adoption, le niveau de la prise de décision, le processus de prise de décision.

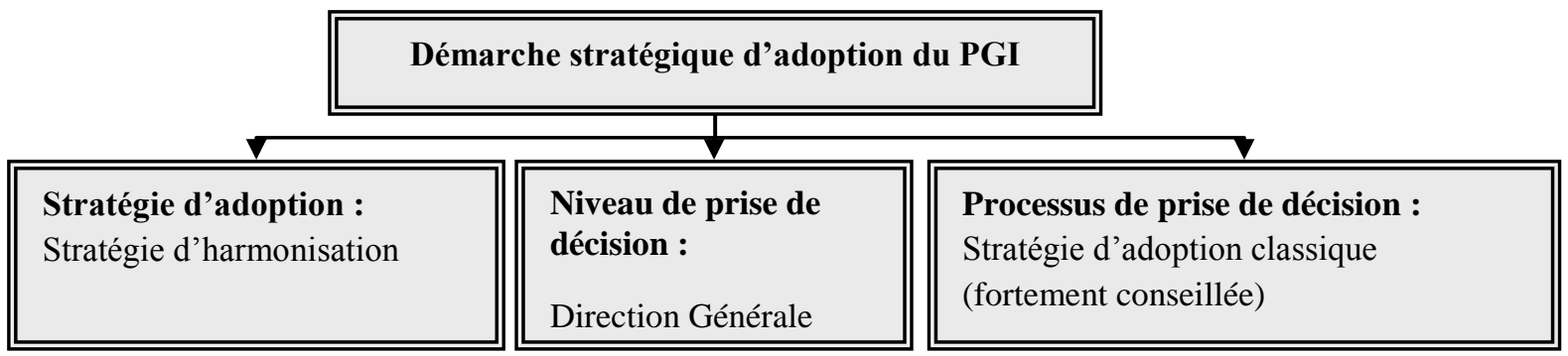

Figure 3 : Démarche stratégique d'adoption du PGI de l'entreprise Alpha

La définition de la vision stratégique d'adoption des PGI est primordiale dans le processus d'intégration d'une solution PGI. Une entreprise qui formule clairement ses objectifs en termes d'adoption saura anticiper et préparer les moyens nécessaires pour les atteindre. Selon le principe de la cohérence stratégique, les choix pris au niveau de l'adoption conditionneront le déroulement du projet dans toutes ses phases.

Notre entreprise a choisi d'adopter son PGI pour des raisons d'harmonisation de son SI avec celui de ses principaux partenaires. D'ailleurs, le projet est une suggestion de l'un de ses partenaires qui détient une participation dans le capital de cette entreprise. La décision est certes prise au niveau de la direction générale de cette entreprise mais sous une forte recommandation de l'un des actionnaires de cette entreprise. Le processus de prise de décision peut par ailleurs être qualifié d'un processus classique avec une stratégie d'adoption fortement conseillée par les partenaires de cette entreprise

\subsection{La démarche stratégique de déploiement du PGI}

La vision stratégique d'une entreprise dans un projet PGI représente son schéma directeur. C'est une variable de différenciation certes, mais qui ne se limite pas à l'étude des choix stratégiques ou des perspectives stratégiques auxquelles aspirent les entreprises. Pour notre recherche, la vision stratégique d'une entreprise intègre ses choix et sa démarche stratégique dans la mise en place de son PGI.

- $\quad$ Stratégie de mise en place accompagnée par un intégrateur

Tous les acteurs de l'entreprise étudiée s'accordent sur la valeur considérable de l'intervention d'un intégrateur dans le projet de mise en place de leur PGI. Au point de considérer le recours à cette aide externe comme une démarche évidente et classique.

Les missions du conseiller intégrateur ont pour objectif commun d'aider les entreprises et organismes clients à se transformer, aussi bien en matière de systèmes d'information et de technologies qu'en matière d'organisation et de management.Au-delà de cet objectif commun, le contexte de chaque client étant unique, chaque mission est spécifique et peut différer assez largement dans le contenu et la nature des compétences mobilisées. Ces missions peuvent déboucher par ailleurs sur des livrables variables. 
Dans le cas de cette entreprise, le recours à un prestataire externe en matière d'intégration du PGI a permis de couvrir plusieurs domaines : La connaissance des meilleures pratiques en termes d'architecture, d'infrastructures et de solutions applicatives, l'accompagnement de mise en œuvre depuis l'élaboration du cahier de charge jusqu'au support de la solution après son déploiement, l'évaluation des risques des solutions préconisées...

Le service d'intégration peut aussi être proposé par la société éditrice du PGI. C'est une nouvelle tendance du marché de l'édition qui dans un objectif de concentration de pouvoir n'hésite pas à s'associer à des cabinets de conseil offrant ce type de prestation ou à des organismes d'intégration spécialisés en la matière. L'avantage pour l'entreprise est avant tout financier, puisque la prestation est facturée moins cher dans une sorte de pack. Cette entreprise a donc à faire à un seul interlocuteur ce qui représente aussi bien un avantage et un inconvénient. Le risque se situe dans l'augmentation du degré de dépendance de cette entreprise vis-à-vis de l'éditeur d'un côté et de manquer l'opportunité d'avoir l'avis objectif d'un intégrateur ayant du recul sur les solutions proposées par l'éditeur.

- Stratégie d'intégration progressive par module ou lot de modules

Sur la base des résultats de cette recherche menée auprès des acteurs concernés par le projet PGI de la PME Alpha, nous avons constaté que l'approche de déploiement de la solution PGI d'un point de vue temporel peut être qualifiée d'intégration progressive par modules ou lot de modules. L'entreprise Alpha a effectivement intégré son PGI de manière séquentielle et partielle par module. Le périmètre fonctionnel s'est vu élargir progressivement au fur et à mesure de l'installation de nouveaux modules. L'installation de chaque module représentait un sous projet démarré séparément et ayant une échéance indépendante.

L'intégration d'un PGI se base essentiellement sur le principe de la transversalité des processus organisationnels. Néanmoins, ce type de déploiement s'élabore dans une logique verticale d'intégration découpant l'organisation en grandes fonctions ou services auxquelles viennent les modules se greffer.

L'approche progressive par module à la différence de celle en Big Bang n'exige pas d'énormes investissements initiaux, par contre elle est maintenue grâce au développement d'interfaces qui servent à assurer la communication entre le nouveau module et l'ancien système en attendant l'intégration de la totalité des modules. Ce qui représente une charge financière supplémentaire au projet.

\section{- Une gestion des écarts stratégiques}

Changer son SI et choisir une solution PGI sont des décisions stratégiques qui vont engager l'entreprise dans un processus de mise en place compresseur. La notion d'écart peut être analysée avant le choix du PGI comme elle peut l'être après le choix. L'introduction de cette notion dans notre analyse provient essentiellement de notre volonté de la mettre en valeur en tant que variable susceptible d'expliquer l'adoption de différentes démarches d'intégration des PGI dans une même entreprise. Toutefois, la confrontation de ce concept au terrain empirique a confirmé son intérêt pour la problématique de recherche notamment pour l'articulation entre le décisionnel et l'opérationnel. La gestion des écarts consiste par ailleurs à l'analyse avant toute prise de décision et à l'évaluation après. Selon les résultats de cette recherche, l'entreprise Alpha reconnait qu'elle n'a pas intégré cette étape dans sa démarche de choix de la solution. Elle justifie ce fait par la nature particulière de cette décision qui a été fortement conseillé par l'un de ses partenaires stratégiques et dont l'objectif principal était d'harmoniser son SI avec celui du partenaire en question. En revanche, l'entreprise avoue avoir procédé à l'analyse des écarts après le choix de la solution; en amont de la phase de déploiement. Généralement, à ce stade du projet, cette démarche sert à qualifier l'ensemble des actions correctives menées par l'entreprise pour réduire les écarts constatés entre les besoins fonctionnels et les propositions de la solution choisie.

Ainsi, lorsqu'une solution est choisie et que la confrontation de sa proposition aux besoins de l'entreprise fait apparaître des écarts stratégiques, deux options peuvent s'offrir à cette entreprise : tenter de les corriger en puisant les possibilités de paramétrage et de développements spécifiques dans le sens d'une spécification de certains modules du PGI et/ou dans le sens d'une standardisation de certains processus; tel est le cas de l'entreprise Alpha. La deuxième option consiste à faire abstraction de ces écarts en décidant de suivre le standard quand même. Une entreprise qui se limite à constater ces écarts sans pour autant essayer de les réduire, a certainement fait la grande partie d'un chemin stratégique judicieux certes, mais qui ne peut être fructueux s'il n'est pas parcouru jusqu'au bout.

\section{- PGI : Outil de Standardisation ou de différenciation de l'entreprise ?}

Les PGI sont désormais des outils de normalisation, de standardisation des processus de gestion. Ils proposent des fonctionnalités fondées sur les meilleures pratiques (Chtioui, 2004) [37]. La question que nous nous sommes posées à ce niveau visait à connaître l'avis des acteurs de l'entreprise Alpha sur ces systèmes en tant qu'outils exclusif de standardisation ou en tant que systèmes offrant des possibilités de différenciation.

La question a fait longuement réfléchir nos interlocuteurs ce qui justifie son intérêt et sa profondeur. Les réponses ont toutes convergé sur le caractère standard qu'imposent ces solutions. Toutefois, une partie des 
réponses reconnait les propriétés standards que peuvent apporter ces solutions mais préconisent leur caractère ouvert à la différenciation. Selon Besson (1999) [38] «L'implantation d'un ERP vise à changer l'organisation. Ce processus est risqué.». L'intégration des PGI constitue donc une refonte du système d'information mais surtout une remise à plat des procédures de gestion au sein de l'organisation. La notion de standardisation a globalement évoqué la même acception chez nos interlocuteurs : elle consiste à faire rentrer les processus existants dans le standard PGI quitte à en éliminer quelques uns et les faire remplacer par d'autres. Le paramétrage est l'un des moyens les plus confirmés pour rentrer dans le standard, toutefois les possibilités de paramétrage offertes par les PGI varient selon la flexibilité de chaque solution. Elles ne sont pas inépuisables, mais l'entreprise a intérêt à les exploiter au maximum avant d'envisager une autre solution comme le développement spécifique. Pour le DSI de l'entreprise Alpha : «L'intégration du 100\% standard peut être un indicateur de différenciation pour l'entreprise : si elle arrive à imposer ce choix à l'ensemble des directions et filiales et se contenter des aspects de paramétrage qui existent c'est qu'elle est différentes des autres ». Adopter le $100 \%$ standard sans aucun développement spécifique correspond à un objectif d'homogénéité qui est l'idéal de beaucoup d'entreprises. Néanmoins, Il est moins évident et assez rarement atteint. Le DSI reconnaît que le système d'information de l'entreprise Alpha est composé en plus du standard PGI d'un certain nombre de développements spécifiques jugés indispensables pour réussir l'intégration du PGI. Autrement dit, ces développements ont servi soit à préserver quelques fonctionnalités spécifiques à l'entreprise soit à compléter la couverture fonctionnelle sur des besoins non couverts par le standard.

De leur côté, les possibilités de paramétrage offertes par le PGI peuvent être considérées à la fois comme un moyen de standardisation et de différenciation. Pour se différencier, une entreprise peut tout simplement adopter une des plusieurs possibilités de paramétrage proposées par le PGI à travers l'alimentation de son système et son usage. Après, il y a selon nos interlocuteurs les services sur lesquels cette entreprise peut se distinguer plus que d'autres tels que les ventes, le marketing, la recherche et développement...qui ne sont pas aisément modélisables par un outil comme peuvent l'être la finance et le contrôle de gestion.

En plus des développements spécifiques qui sont les moyens les plus utilisés par les entreprises pour adapter le PGI à leurs spécificités, une entreprise peut se différencier en ayant recours à différents outils et applications informatiques à côté du PGI. L'entreprise évite ainsi le modèle unique proposé par le PGI et tente d'enrichir son système d'information tout en apportant une réponse aux adaptations-marché.

\section{Les démarches d'intégration organisationnelle du PGI et leurs implications organisationnelles}

Sur la base de ces variables stratégiques analysées particulièrement au niveau de chaque fonction supportée par un module du PGI, nous avons pu distinguer trois démarches d'intégration adoptée par les fonctions de l'entreprise de notre recherche. Elles se présentent ainsi :

\subsection{Intégration spécifique}

Une logique dont la tendance principale préconise un besoin d'adaptation du module du PGI aux besoins du service ou de la fonction correspondante. Cette adaptation consiste dans des opérations de configuration qui dépendent des possibilités de paramétrages offertes par la solution. Lorsque ces possibilités sont épuisées sans que le service en question en soit satisfait, ce dernier peut décider de faire des spécifiques à côté du module, de ne pas intégrer le module définitivement et d'en faire un gros spécifique. La non-intégration d'un module peut par ailleurs être considérée comme un aspect de l'intégration spécifique. Les raisons de la non-intégration peuvent être liées à la non couverture du besoin par le module ou la non adéquation de la solution avec les besoins du service en question. L'adoption de cette configuration dépend du métier ou du flux concernés par l'intégration et de la mentalité des acteurs impliqués dans le projet. Les besoins et les objectifs stratégiques varient en passant d'un service à un autre. Ce qui a été constaté chez l'entreprise enquêtée c'est que l'objectif des services adoptant une intégration spécifique (service commercial et service Marketing) consiste certes à changer le système en place, mais sans pour autant bouleverser l'organisation habituelle du travail. Elle vise ainsi à remplacer l'ancien système par un nouveau qui est vaste et qui demande un travail de spécification pour l'adapter aux besoins fonctionnels. Les adeptes de cette configuration peuvent avoir une vision déterministe (délibérée ou non) du changement en considérant la technologie comme un support voire un résultat de la stratégie de l'entreprise. La mentalité des acteurs concernés par l'intégration peut aussi impacter la démarche d'intégration. Les "gens" de la production ne réagissent pas de la même façon que les commerciaux et les "Marketers" devant un projet d'implémentation d'un PGI. L'esprit cartésien des ingénieurs de production pose moins de complication à l'intégration en termes de résistances aux changements. Ils savent précisément ce qu'ils veulent et jugent le projet sur ses résultats. Tandis que les commerciaux sont plus exigeants au niveau du respect des spécificités de leurs métiers et plus résistants aux changements survenus à leurs méthodes de travail ce qui requiert plus d'effort pour les convaincre à adhérer au projet selon l'avis du chef de projet PGI. 


\subsection{Intégration standard}

C'est la grande tendance du projet PGI de l'entreprise Alpha. Il s'agit d'une logique qui préconise que techniquement parlant, le PGI devraient l'emporter sur tous les autres aspects d'implémentation. Il s'agit d'une vision déterministe du projet et des changements attendus. En effet, la technologie est là pour piloter, le reste doit suivre. L'organisation est censée être plus flexible que le PGI. Elle doit s'aligner par ailleurs aux standards proposés par le nouveau système en termes d'architecture, de procédures et de règles de gestion...

Le PGI selon cette approche est un outil qui offre tellement de possibilités de paramétrage que l'entreprise peut éventuellement se passer des développements spécifiques. La notion d'écart stratégique dans ce type d'intégration est présente mais de façon passive. Cela veut dire que l'existence de cet écart n'empêche pas l'entreprise, sous l'approbation de son éditeur et intégrateur, d'en faire abstraction pour pencher vers la solution standard. L'écart entre le besoin fonctionnel de l'organisation et la réponse de couverture proposée par la solution se voit donc s'effacer dans le respect total du standard. Les métiers qui supportent ce type d'intégration dans le cas de notre étude sont des métiers classiques (la gestion des achats par exemple) même s'ils peuvent afficher des spécificités fonctionnelles différentes du standard, la tendance standardisante l'emporte à la fin. Et plus l'entreprise est normalisée plus elle va vers le standard. Cela peut paraître paradoxal, mais cette standardisation peut être considérée comme une source de différenciation. En effet, sur un marché concurrentiel, une entreprise qui réussit une intégration $100 \%$ standard (ce qui est rare) est une entreprise distincte. Peu d'éditeurs de PGI peuvent se vanter de proposer dans leurs solutions des réponses à l'ensemble des besoins et spécificités des différentes entreprises. Quand on voit le mal que se donnent les solutions verticalisées (PGI métier) à le faire, on peut conclure que c'est perdu d'avance pour les solutions généralisées.

\subsection{Intégration de compromis}

Comme sa dénomination l'indique, cette tendance se base sur une approche compromiteuse entre le besoin et le standard. Dans ce type d'intégration il est rarement question d'écart. Le standard est censé réunir l'ensemble des meilleures pratiques dont l'entreprise a besoin et même celles dont elle a n'a pas pressenti le besoin. Par ailleurs, le PGI peut non seulement couvrir les besoins fonctionnels existants mais en créer d'autres. Les services ou entités les plus concernées par ce type d'intégration sont généralement des services dont les procédures sont tellement travaillées et standardisées qu'ils présentent rarement des besoins spécifiques non couverts par la solution. On parle souvent de service comptable et financier. Les normes comptables sont connues en général que ce soit au niveau local, national ou international. Certains éditeurs (comme SAP) ont misé sur cette partie financière qu'elle en est devenue leur argument de vente.

Pour intégrer son PGI selon une démarche de compromis, l'entreprise réorganise des procédures, retranscrit ce qui colle bien, et fait un compromis entre ce qu'elle a et ce qui se fait dans le PGI en ayant recours le plus souvent aux possibilités de paramétrage offertes par le standard. Toutefois, dans ce type d'intégration il s'agit essentiellement de compromis entre système et organisation, entre les différents acteurs de l'entreprise : utilisateurs, utilisateurs clés, DG, intégrateurs...et entre paramétrage et développements spécifiques. Toutefois, cette notion de compromis est une conséquence d'une planification en amont du projet et qui vise à profiter au maximum de toutes les fonctionnalités du standard.

\section{L'intégration du PGI et le changement organisationnel}

Nous avons tenté dans cette recherche d'aborder le changement organisationnel en tant que paramètre indissociable de l'étude de la question de l'intégration organisationnelle des PGI. Il permet, en effet, d'étudier les implications de cette intégration sur plusieurs niveaux : financier, humain et organisationnel. Il permet également de se rendre compte de la complexité de ce type de projet à travers l'étude des difficultés de leur mise en place. Enfin, il permet d'analyser la démarche adoptée par l'entreprise afin de gérer les changements liés à ce type de projet.

\subsection{Apports du PGI}

Plusieurs études ont porté sur l'analyse de l'impact des systèmes PGI sur les organisations (Vemuri et Palvia, 2006; Velcu, 2007) [39]. Selon les résultats de ces recherches, les systèmes PGI permettent aux organisations de réaliser des économies d'échelles, de réduire les coûts généraux et administratifs, de réduire le temps des différents processus organisationnels, d'assurer une meilleure rotation des stocks, de faciliter la communication et la collaboration inter-organisationnelle...(O’leary, 2000 ; Booth et al, 2000, Markus et Tanis, 
1999) [40].Nous avons fait le choix à travers cette recherche d'analyser la nature des impacts liés à la mise en place d'un ERP dans une PME Marocaine. Nous avons procédé dans un premier temps par l'étude de ce que l'intégration d'un PGI peut impliquer comme conséquences positives sur l'entreprise et son organisation, avant d'analyser les aspects négatifs liés à ce projet (difficultés et limites) Nous avons essayé à travers les résultats de l'étude de situer les apports des PGI aux niveaux financier, organisationnel et humain de l'entreprise enquêtée (Fig.4). Le but étant de donner aux décideurs et acteurs organisationnels une image réaliste des attributs positifs de ces solutions.

\begin{tabular}{|c|c|c|}
\hline & \multicolumn{2}{|l|}{ Apports du PGI } \\
\hline$\nabla$ & & 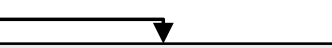 \\
\hline Niveau financier & Niveau organisationnel & Niveau humain \\
\hline$\frac{1}{1}$ & 7 & +1 \\
\hline $\begin{array}{l}\text {-Optimisation des } \\
\text { stocks et des flux de } \\
\text { gestion } \\
\text {-Amélioration de la } \\
\text { traçabilité des } \\
\text { produits et la qualité } \\
\text { des données } \\
\text {-Gain de temps } \\
\text {-Economies d'échelle } \\
\text {-Réduction des coûts } \\
\text { d'exploitation } \\
\text {-Réduction des coûts } \\
\text { cachés de gestion } \\
\text {-Gains de productivité } \\
\text {-Amélioration de la } \\
\text { profitabilité de } \\
\text { l'entreprise } \\
\text {-Amélioration du retour } \\
\text { sur investissement. }\end{array}$ & $\begin{array}{l}\text {-Amélioration des processus et des règles de gestion } \\
\text { - La production en temps réel des informations } \\
\text { utiles et de qualité } \\
\text { - Une gestion transparente des activités } \\
\text {-Une amélioration des processus de prise de } \\
\text { décision } \\
\text {-Définition des rôles et responsabilités des acteurs } \\
\text {-Intégration de l'ensemble de la chaîne de valeur } \\
\text {-Mise en place des processus de contrôle au long de } \\
\text { la chaîne de valeur } \\
\text {-Rapprochement interne des différentes fonctions de } \\
\text { l'entreprise } \\
\text {-Rapprochement externe avec les partenaires de } \\
\text { l'entreprise } \\
\text {-Amélioration de la qualité d'image de l'entreprise } \\
\text {-Amélioration de la capacité d'attraction de } \\
\text { nouveaux clients }\end{array}$ & $\begin{array}{l}\text {-Satisfaction des } \\
\text { acteurs concernés } \\
\text { par le projet } \\
\text {-Création de } \\
\text { nouveaux postes de } \\
\text { travail } \\
\text {-Evolution des } \\
\text { contenus des postes } \\
\text { de l'exécutif au } \\
\text { décisionnel } \\
\text {-Responsabilisation } \\
\text { des acteurs et } \\
\text { augmentation de } \\
\text { leur rigueur. } \\
\text {-Transfert de pouvoir } \\
\text { des informaticiens } \\
\text { aux utilisateurs. }\end{array}$ \\
\hline
\end{tabular}

Figure 4 : Les Apports du PGI de l'entreprise Alpha sur le plan financier, humain et organisationnel.

\subsection{Difficultés des projets PGI}

Pour ne pas s'arrêter aux exploits des PGI, nous avons souhaité analyser la partie sombre de leurs projets d'intégration. L'expérience de l'entreprise enquêtée nous a permis de faire une longue liste des difficultés rencontrées à l'occasion de ce projet. Nous les avons analysées et classifiées au fur et à mesure de l'avancement du projet PGI. Nous avons pu par ailleurs relever trois grands types de difficultés : difficultés d'avant projet, difficultés de démarrage et difficultés d'après projet (Fig.5).L'intérêt de ce travail pour les décideurs et acteurs projets consiste à les sensibiliser vis à vis de la complexité des projets PGI et des risques qu'ils peuvent éventuellement encourir. Les entreprises qui sont entrain de mettre en place un PGI ou qui souhaitent le faire un jour sauront ainsi à quoi s'attendre et chercheront les moyens pour anticiper voire éviter ces difficultés et limiter les risques qui y sont associés. 


\begin{tabular}{|c|c|c|c|}
\hline \multicolumn{3}{|r|}{ Difficultés du projet PGI } & \multirow{3}{*}{ Difficultés d'après projet } \\
\hline \multicolumn{2}{|c|}{ Difficultés d'avant projet } & Diffioultós dr dómonnom & \\
\hline & & & \\
\hline $\begin{array}{l}\text {-Au niveau de la } \\
\text { phase préparation } \\
\text { et analyse de } \\
\text { projet } \\
\text { - Au niveau des } \\
\text { choix de } \\
\text { méthodologies } \\
\text { d'adoption et de } \\
\text { déploiement } \\
\text { - Au niveau de } \\
\text { l'implication du } \\
\text { facteur humain } \\
\text { dans le projet } \\
\text { - Au niveau de la } \\
\text { phase de reprise } \\
\text { de données }\end{array}$ & $\begin{array}{l}\text {-D'o } \\
\text { après } \\
\text { factu } \\
\text { fourr } \\
\text { prod } \\
\text {-D'o } \\
\text { des a } \\
\text { finau } \\
\text { proje } \\
\text { utilis } \\
\text {-D'o } \\
\text { diffé } \\
\text { proje } \\
\text { diver } \\
\text { fonct }\end{array}$ & $\begin{array}{l}\text { technique : flottement général juste } \\
\text { lémarrage, retards de traitement de } \\
\text { perturbation de paiement des } \\
\text { urs, arrêts partiel ou complet de } \\
\text { on... } \\
\text { humain : manque d'implication } \\
\text { Irs (utilisateurs clés, utilisateurs } \\
\text { G), départ des acteurs en plein } \\
\text { ficits en formation des } \\
\text { Irs... } \\
\text { organisationnel : engagement de } \\
\text { partenaires à l'occasion du même } \\
\text { vergence d'intérêt des partenaires, } \\
\text { ce d'objectifs des différentes entités } \\
\text { eelles concernées par le projet. }\end{array}$ & $\begin{array}{l}\text {-Difficultés sociales : résistances aux } \\
\text { changements, suppression de postes, baisse des } \\
\text { bras de certains acteurs après le démarrage, } \\
\text { difficultés de reprise d'une activité normale après } \\
\text { la dissolution de l'équipe projet, conflits de } \\
\text { pouvoirs } \\
\text {-Difficultés techniques : relatives aux montées de } \\
\text { versions, aux maintien des interfaces liant les DS } \\
\text { aux anciennes versions, sous exploitation des } \\
\text { PGI, demandes d'adaptation non justifiées par } \\
\text { les utilisateurs et mal comprises par les centres } \\
\text { supports. } \\
\text {-Difficultés organisationnelles : dérives } \\
\text { incompréhensibles au niveau des coûts et du } \\
\text { temps alloués au projet, évaluations précoces des } \\
\text { résultats du projet, manque de recul de } \\
\text { l'entreprise }\end{array}$ \\
\hline
\end{tabular}

Figure 5 : Difficultés rencontrées par l'entreprise Alpha avant, pendant et après le projet PGI

\subsection{Les limites du PGI}

La question des limites du PGI n'est qu'un enchaînement naturel dans le processus de compréhension des décisions et choix faits par les décideurs hiérarchiques à l'occasion du projet PGI. La question des limites des PGI est souvent liée dans la littérature spécialisée aux facteurs d'échec de ces projets (Davenport, 1998 ; Rao, 2000 ; Themistocleous et al, 2001...) [41]. Dans ce sens, les limites techniques ou organisationnelles des PGI peuvent être considérées comme des facteurs qui contribuent à l'échec partiel ou total d'un projet PGI (Rao, 2000) [42] mais qui ne l'expliquent pas systématiquement. Cette recherche était pour nous l'occasion de recueillir l'avis des acteurs concernés par le projet PGI de l'entreprise Alpha sur la question des limites de ce dernier. Leurs réponses ont témoigné d'une prise de conscience générale de ce que peut être les limites du PGI en tant qu'approche, en tant qu'outil et en tant que projet (Fig.6). L'analyse de ces limites peut fournir aux décideurs dans d'autres entreprises des éléments d'éclairage et d'aide aux décisions relatives notamment au choix de l'approche intégrée et de la solution en question.

\begin{tabular}{|c|c|c|}
\hline & Limites du PGI & \\
\hline Limites de l'outil PGI & Limites du projet PGI & Limites de l'approche intégrée \\
\hline 7 & $\overline{7}$ & $\bar{v}$ \\
\hline $\begin{array}{l}\text {-Rigidité de fonctionnement } \\
\text {-Rigidité de manipulation } \\
\text {-Imposition de formes } \\
\text { d'organisation préfabriquées } \\
\text {-Rétrécissement des marges } \\
\text { d'autonomie des entreprises } \\
\text {-Augmentation de la pression, } \\
\text { du stress, et de la frustration } \\
\text { des utilisateurs dans le travail } \\
\text { auntidien }\end{array}$ & $\begin{array}{l}\text {-Investissement financier et } \\
\text { humain faramineux } \\
\text {-Projet lourd et complexe } \\
\text {-Projet risqué } \\
\text {-Compétences humaines rares et } \\
\text { coûteuses } \\
\text {-Mouvements de fusion- } \\
\text { acquisition entre éditeurs nuit le } \\
\text { déroulement des projets }\end{array}$ & $\begin{array}{l}\text {-Ouverture limitée de la } \\
\text { solution et enfermement de } \\
\text { l'entreprise } \\
\text {-Dépendance vis-à-vis de } \\
\text { l'éditeur } \\
\text {-Mise en place dépassée : } \\
\text { base de données + Serveurs } \\
\text {-Nouvelles propositions } \\
\text { technologiques SOA }\end{array}$ \\
\hline
\end{tabular}

\subsection{Le management de changement}

Figure 6 : Limites du PGI selon la perception des acteurs de l'entreprise Alpha

Parler de changement organisationnel amène à s'interroger sur les voies qui favorisent sa réalisation. A ce titre, L'apprentissage organisationnel (Métais, Roux-Dufort, 1997) [43] se présente comme l'une des voies possibles pour une réalisation aboutie du changement. Selon Bernier et al. (2003) [44] « Le changement requiert l'apprentissage et la maîtrise non seulement de nouvelles compétences technologiques, mais, dans bien des cas, de nouvelles compétences professionnelles, relationnelles et interpersonnelles »En revanche, des phénomènes de résistances (Besson, 1999) [45] peuvent surgir à l'occasion de ce type de projet. Une gestion a posteriori (Lapointe \& Rivard, 2005) [46] de ces phénomènes limiterait sans doute son efficacité. En effet, la gestion des changements induits par l'implémentation d'un PGI peut être qualifiée d'un projet dans ou en parallèle du projet 
PGI. Il consiste avant tout à un travail d'anticipation, d'information, de formation et d'implication des utilisateurs dans le projet. Il s'agit de mettre l'utilisateur au centre du processus du changement en accordant une attention particulière à ses réactions pendant tout le projet (Markus, Axline, Petrie, Tanis 2000)[47].Nous avons cherché à analyser la tendance générale adoptée par l'entreprise enquêtée pour la gestion des changements associés à son projet PGI. Cette entreprise considère le management du changement comme un moyen pour accompagner les changements dus aux projets PGI. C'est un rouleau compresseur que l'entreprise à essayé de gérer au fur et à mesure de l'avancement du projet PGI. Il s'agit d'un ensemble d'actions que l'entreprise a été disposée à mobiliser pour faire face à l'ensemble des transformations et évolutions susceptibles de bouleverser l'ordre organisationnel. Des actions de communication ont donc été prévues ainsi que la formation des utilisateurs clés et finaux avant et pendant le projet, L'entreprise intègre l'activité de support aux utilisateurs du système comme une action qui aide à la gestion des changements. A la différence de la dimension globale des projets de "Change Management", l'accompagnement du changement dans le cas de cette entreprise substitue la notion d'anticipation du changement et des comportements des acteurs face au nouveau PGI au principe selon lequel les acteurs ont toujours besoin de temps pour apprécier l'outil et se l'approprier à travers l'usage (Le Goaziou, 1992) [48]. Leur motivation provient essentiellement de leur satisfaction à l'usage.

\section{CONCLUSION}

Les PGI sont des outils d'intégration de l'organisation. Un constat tant affirmé dans la littérature experte. Toutefois, la complexité de la mise en place des progiciels intégrés explique la difficulté des entreprises à améliorer leur existant en renforçant l'intégration de leur gestion via les PGI. Dans la définition que nous avons attribuée au concept de l'intégration organisationnelle, nous avons insisté sur la notion de "recherche permanente d'équilibre » entre l'intégration du standard et le développement des spécifiques. Les résultats de la recherche ont démontré que cette notion d'équilibre ne signifie pas forcément la parité 50/50 entre paramétrage du standard et développement du spécifique dans les projets PGI. Une entreprise peut trouver son équilibre d'intégration au moment où l'ensemble de ses besoins fonctionnels ait été couvert et de manière à satisfaire les différentes parties impliquées dans le projet. Ainsi, en adoptant une intégration standard par exemple, une fonction d'entreprise peut trouver son équilibre essentiellement dans le paramétrage avec un nombre négligeable de développements spécifiques. Et inversement, l'équilibre pour une autre fonction, qui intègre un PGI en voulant garder la même structure et les mêmes procédures, peut se définir par un nombre élevé de spécifiques. Tout est question d'équilibre : Equilibre entre l'offre de la solution et les besoins fonctionnels de l'entreprise, équilibre entre la standardisation des processus et le développement des spécifiques, et équilibre entre la politique globale de l'entreprise et les stratégies fonctionnelles de ses services. Concernant la particularité de l'intégration organisationnelle des PGI dans une PME, il semble qu'une grande entreprise et une PME ont en général les mêmes problématiques managériales. D'autant plus si elles opèrent dans le même secteur d'activité, l'industrie par exemple. Ce qui peut les différencier à ce niveau en particulier ce serait le degré de complexité de ces problématiques et le nombre de variables à prendre en compte pour les définir. Les processus de gestion d'une grande structure sont caractérisés par une plus grande transversalité, un nombre plus élevé d'acteurs concernés, un processus de prise de décision plus lent et moins flexible que celui d'une PME. Ce qui peut justifier, ne serait-ce que partiellement, qu'une grande entreprise met souvent plus de temps et de ressources pour intégrer son PGI qu'une PME. En revanche, au niveau de la démarche d'intégration, la présente recherche montre bien l'existence de différentes modalités d'intégration d'un PGI qui peuvent être adoptées par les différentes fonctions d'une même entreprise. Ce qui peut distinguer finalement une grande entreprise d'une PME en matière d'intégration d'un PGI, c'est l'orientation stratégique globale de ces entreprises en général et les stratégies spécifiques à chaque domaine fonctionnel qui fait l'objet d'une intégration modulaire. Dans une grande entreprise comme dans une petite entreprise, les différentes fonctions peuvent adopter le même schéma d'intégration ou des schémas différents. Ce qui est attendu c'est que le résultat de leur intégration correspondrait le plus souvent à un schéma mixte : une sorte de combinaison des trois modalités d'intégration distinguées principalement dans cette recherche, à savoir ; l'intégration spécifique, l'intégration standard et l'intégration de compromis. Néanmoins, il est important d'évoquer que certaines limites sont attribuées à notre recherche notamment sur le plan empirique. En effet, nous nous sommes limités à une étude de cas d'une PME marocaine ayant un PGI en place. Les résultats de cette recherche analysent bien l'existence de différentes modalités d'intégration adoptées par les différentes fonctions de cette entreprise ainsi que les implications de cette intégration sur le plan organisationnel. Il est donc primordial d'élargir notre échantillon de la recherche dans l'ambition de produire des résultats généralisables.

\section{BIBLIOGRAPHIE}

[1] B. Vincent et S. Gharbi, Impact du déploiement de SAP R/3 sur la performance globale d'une entreprise et facteurs clés de succès: proposition d'un tableau de bord et application dans le secteur de l'industrie pharmaceutique, Journée de recherche à l'IAE de Montpelier, 2004, 1-27.

[2] S. Mourlon et L. Neyer, Tout ce que nous avons voulu savoir sur les ERP, Les Annales des Mines de Paris, 2002. 
[3] N. Bancroft, Implementing SAP R/3 (Manning Publications, Greenwich, 1996)

E. Brynjolfsson et L. M. Hitt, Beyond Computation: Information Technology, Organizational Transformation and Business Performance, Journal of Economic Perspectives, 2000, 14(4), 23-48

W. Gu et S. Gera, The Effect of Organizational Innovation and Information Technology on Firm Performance, The Canadian Economy in Transition Series - Statistics Canada, 2004.

B. Dostie et R. Jayaraman, Organizational Redesign, Information Technologies and Workplace Productivity, Les Cahiers du CREF, 2008.

[4] J.L. Lequeux, Manager avec les ERP, progiciels de gestion intégrés et internet (Les Editions d'Organisations, Paris, 1999).

[5] R. El Amrani, Vision organisationnelle cible comme facteur de réussite d'un projet ERP: le cas SAP chez l'entreprise Consto, 8 ème colloque de l'AIM, Grenoble, 2003.

[6] S. Baile et R. Lesuisse, De la spécificité à la généricité des logiciels, dans Vuibert (éd.) Faire de la recherche en SI, (coordonné par F.Rowe, 2002).

[7] O.Hanseth et K. Braa, Technology as a traitor : emergent SAP infrastructure in a global organization, International conference on information system, Helsinki, 1998.

E.E. Watson, H. Scneider et E.J. Ourso, Using ERP system in education, communication of the association for information systems, 1999 , vol1, $\mathrm{n}^{\circ} 9$.

[8] W. Gu et S. Gera, The Effect of Organizational Innovation and Information Technology on Firm Performance, The Canadian Economy in Transition Series - Statistics Canada, 2004.

B. Dostie et R. Jayaraman, Organizational Redesign, Information Technologies and Workplace Productivity, Les Cahiers du CREF, 2008.

[9] R. Reix, Systèmes d'information et management des organisations, (Paris, Vuibert, 2000).

[10] N.H. Bancroft, H. Seip et A. Sprengel, 1997, Implementing SAPR/3: How to Introduce a Large System into a Large Organization (Manning, Greenwich, 1997).

T.H. Davenport, Putting the enterprise into the enterprise system, Harvard Business Review, 1998, p 121-131.

M. Grandlund et T. Malm 2000, Some empirical evidence of the effects of ERP-systems on management accounting, Cahier de recherche de Turku School of Economics and Business Administration, Finlande, 2000.

[11] S. Baile et R. Lesuisse, De la spécificité à la généricité des logiciels, dans Vuibert (éd.) Faire de la recherche en SI, (coordonné par F.Rowe, 2002).

[12] E.E. Watson, H. Scneider et E.J. Ourso, Using ERP system in education, communication of the association for information systems, 1999, vol 1, n 9 .

[13] O.Hanseth et K. Braa, Technology as a traitor : emergent SAP infrastructure in a global organization, International conference on information system, Helsinki, 1998.

[14] AFSCET : Association Française des Sciences des Systèmes Cybernétiques, Cognitifs et Techniques.

G. Donnadieu, D. Durand, D. Neel, E. Nunez, L. Saint-Paul, L'Approche systémique : de quoi s'agit-il ? Synthèse des travaux du Groupe AFSCET (Diffusion de la pensée systémique), 2003.

[15] J.J Lapointe, La conduite d'une étude de besoins en éducation et en formation : une approche systémique (Presses de l'université du Québec, 1992).

[16] P. Checkland, 1976, Science and the systems paradigm, Internationnal of general system,1976, vol 3.

P. Checkland, System thinking, system practice (John Wiley\& Son, London, 1981).

B. Commoner, L'encerclement (Paris, traduit de l'américain par G. Durant, Edition du Seuil,1972).

P. Watzlawick, Le langage du changement, Eléments de communication thérapeutique (Traduit de l'américain par Wienner-Renucci et Bansard, Paris, Seuil, 1980).

[17] J.L. Le Moigne, La théorie du système général, théorie de la modélisation (Paris : col. Systèmes-décisions, Presse Universitaires de France, 1977).

[18] L.von. Bertalanffy, General systems theory, in: General systems, Yearbook of the Society for General Systems Research, vol. 1, 1956.

[19] E. Morin, Science avec conscience (Paris, Fayard, 1982).

[20] J.L. Le Moigne, La théorie du système général, théorie de la modélisation (Publication de l'édition, 1994).

[21] K. Boulding, General Systems theory, The skeleton of science, Management Science, Avril 1956

[22] L.von. Bertalanffy, General System Theory: Foundations, Development, Applications (Revised edition, New York: George, 1968).

[23] F. Deltour et E. Vaast, Quand technologie et organisation construisent un réseau d'échanges professionnels : une étude de cas structurationniste, Cinquième congrés de l'AIM, montpellier, Novembre 2000.

[24] L.von. Bertalanffy, Théorie générale des systèmes ( Paris, Dunod,1973).

[25] M. Kalika, 2003, TIC et stratégie, dans Editions Liaisons, Le E-management: quelles transformations dans l'entreprise ? ( Paris, p. 71).

[26] A. Rallet, Télécommunication et compétitivité, (Réseaux N54, CENT, 1992).

R.L. Daft et R.M. Lengel, Organization information requirements, media richness and structural design, Management science, 1986, volume $52, n^{\circ} 5$.

[27] G.B. Davis, Management Information Systems: Conceptual Fondations. Structure, and Development (McGraw Hill, New-York, 1974).

[28] E.B. Swanson et N.C. Ramiler, The organization vision in information system innovation, Organization science, 1997, vol 8, n5, p 458-474. 
R. El Amrani, F. Rowe, M. Bidan, B. Geffroy-Marronat, R. Marciniak, Déploiement des PGI, transversalité et facteurs clés du changement, actes de la conférence pré-ICIS Journée de la recherche francophone en systèmes d'information, 2002, Barcelone.

[29] R. El Amrani, F. Rowe, M. Bidan, B. Geffroy-Marronat, R. Marciniak, Déploiement des PGI, transversalité et facteurs clés du changement, actes de la conférence pré-ICIS Journée de la recherche francophone en systèmes d'information, 2002, Barcelone.

[30] P. Senge, The Leader's New Work : Building Learning Organizations, Sloan Management Review, Fall, 1990.

[31] S. Change et G.G Gable, A comparative analysis of major ERP lifecycle implementation, management and support issues in Queensland government, Pacific Asia conference on information systems, 2001.

V. Kale 2000, Implementing SAP R/3 the guide for business and technology managers (Indianapolis, IN:Sams Publishing, 2000).

K. Lyytinen, L. Mathiassen et J. Ropponen, Attention shaping and software risk : A categorical analysis of four classical risk management approaches, Information systems research, 1998.

W.L. Soh, S.S. Kien, J. Tay-Yap, Cultural fits and misfits: is ERP a universal solution? Communication of the ACM, 2000 .

[32] J. Henderson et N. Venkatraman, Strategic alignment: Leveraging information technology for transforming organizations, IBM Systems Journal, 1993.

J.N. Luftman, Competing in the Information Age (Oxford University Press, 1996)

Y. Chan et S. Huff, Strategic information systems alignment, Business Quarterly, 1993.

B.H. Reich et I. Benbasat, Measuring the linkage between business and information technology objectives, MIS Quarterly, 1996.

[33] Venkatraman N, Reconfigurations d'entreprises provoquées par les technologies de l'information, in Scott Morton, L'entreprise Compétitive au Futur (Les Editions d'Organisation, 1995) p 151-195.

[34] F.X. Vaujary, Du management stratégique des NTIC au management stratégique de l'appropriationdes NTIC, 2002,Online: http://www.bibliotheques.univlilles.fr/grisemine.

[35] M. Marchesnay, Management stratégique ( les éditions de l'ADREG, 2004)

[36] H.I. Ansoff, Corporate Strategy (McGraw-Hill, 1965).

[37] T. Chtioui, Les effets d'une normalisation des processus de gestion, 25ème Congrès de l'Association Francophone de Comptabilité (AFC) - Normes et mondialisation, 2004.

[38] P. Besson, Les ERP à l'épreuve de l'organisation, ERP/PGI et changement, Systèmes d'information et management, 1999, vol. $4, n^{\circ} 4$

[39] V.K. Vemuri, et S.C. Palvia, Improvement in Operational Efficiency Due to ERP Systems Implementation: Truth or Myth? Information Resources Management Journal, 2006, 19(2), 18-36.

O. Velcu, Exploring the Effects of ERP Systems on Organisational Performance: Evidence from Finnish Companies, Industrial Management \& Data Systems, 2007, 107(9), 1316-1334.

[40] D.E. O’leary, Enterprise Resource Planning Systems : Systems, life cycle, Electronic commerce, and Risk (Cambridge University press, 2000).

P. Booth, Z. Matolcsy et B. Wieder, Integrated Information Systems (ERP-systems) and accounting practice - The Australian Experience, Third European Conference on Accounting Information Systems, Munich, 2000, Germany.

M.L. Markus et C. Tannis, The enterprise systems experience-from adoption to success, in R.W. Zmud (ed, Fraing the domains of IT research), Glimpsing the future through the past (Cincinnati, OH: Pinnaflex Educational Resources, 1999, Inc, 173-207).

[41] T.H. Davenport, Putting the enterprise into the enterprise system, Harvard Business Review,1998, July - Auguest, 121- 133.

S.S. Rao, Enterprise Resource planning: business needs and technologies", Industrial Management \& Data Systems, 2000, Vol. 100.

M. Themistocleous, Z. Irani et R. O'Keefe, ERP and application integration: exploratory survey, Business Management Journal, 2001, 7(3), pp. 195-204

[42] S.S. Rao, Enterprise Resource planning: business needs and technologies", Industrial Management \& Data Systems, 2000, Vol. 100.

[43] E. Métais et C. Roux-Dufort, Vision stratégique et formes d'apprentissage organisationnel : des stratégies d'adéquation aux stratégies d'intention, Sixième Conférence Internationale de Management Stratégique, AIMS, 1997, HEC Montréal.

[44] C. Bernier, C. Bareil et A. Rondeau, Transformer l'organisation par la mise en œuvre d'un ERP : une appropriation à trois niveaux, Gestion, 2003, vol. 27, n 4, p.24-33.

[45] P. Besson, Les ERP à l'épreuve de l'organisation, Systèmes d'Information et Management, 1999, vol. 4, nº4.

[46] L. Lapointe et S. Rivard, A Multilevel Model of Resistance to Information Technology Implementation", MIS Quarterly, 2005, vol.29, $\mathrm{n}^{\circ} 3$.

[47] M.L. Markus, S. Axline, D. Petrie, C. Tanis, Learning from adopters' experiences with ERP: problems encountered and success achieved, Journal of Information Technology, 2000, vol. 15.

[48] V. Le Goaziou, Usages et usagers : un travail de convergence, dans L'Harmattan, Ces réseaux que la raison ignore, (Centre de Sociologie et de l'Innovation, Paris, 1992, P153-168). 